\title{
The universality of equivariant complex bordism
}

\author{
Michael Cole $^{1}$, J.P.C. Greenlees ${ }^{2}$, I. Kriz ${ }^{3}$ \\ 1 Department of Mathematics, Hofstra University, Hempstead, NY 11549, USA \\ (e-mail: matmzc@hofstra.edu) \\ 2 School of Mathematics and Statistics, Hicks Building, Sheffield S3 7RH, UK \\ (e-mail: j.greenlees@ sheffield.ac.uk ) \\ 3 Department of Mathematics, University of Michigan, Ann Arbor, MI 48109, USA \\ (e-mail: ikriz@math.lsa.umich.edu)
}

Received: 14 February 2000; in final form: 4 August 2000 /

Published online: 19 October 2001 - (c) Springer-Verlag 2001

\begin{abstract}
We show that if $A$ is an abelian compact Lie group, all $A$ equivariant complex vector bundles are orientable over a complex orientable equivariant cohomology theory. In the process, we calculate the complex orientable homology and cohomology of all complex Grassmannians.
\end{abstract}

\section{Introduction}

Suppose $A$ is an abelian compact Lie group. We recall [3] that an $A$ equivariant cohomology theory $E_{A}^{*}(\cdot)$ is orientable if complex line bundles are well behaved. More precisely, we let $\mathbb{C} P(\mathcal{U})$ denote the space of lines in a complete complex $A$-universe $\mathcal{U}$, and $\epsilon$ denote the trivial representation. We say that $E_{A}^{*}(\cdot)$ is a complex stable ring theory if there are suspension isomorphisms

$$
\sigma_{V}: \tilde{E}_{A}^{n}(X) \stackrel{\cong}{\longrightarrow} \tilde{E}_{A}^{n+|V|}\left(S^{V} \wedge X\right)
$$

for all complex representations $V$, where $S^{V}$ is the one-point compactification of $V$, and $|V|$ is the real dimension of $V$; these are required to be transitive, and given by multiplication with a generator of $\tilde{E}^{|V|}\left(S^{V}\right)$. We say the theory is complex orientable if in addition, there is a cohomology class $x(\epsilon) \in E_{A}^{*}(\mathbb{C} P(\mathcal{U}), \mathbb{C} P(\epsilon))$ which restricts to a generator of

$$
E_{A}^{*}(\mathbb{C} P(\alpha \oplus \epsilon), \mathbb{C} P(\epsilon)) \cong \tilde{E}_{A}^{*}\left(S^{\alpha^{-1}}\right)
$$

The third author acknowledges support from an NSF National Young Investigator award. 
for all one dimensional representations $\alpha$. Thus if the complex orientation $x(\epsilon)$ is in cohomological degree 2 , it determines a complex stable structure. Many important theories are complex orientable, for instance equivariant $K$ theory, tom Dieck's equivariant bordism $M U_{A}^{*}(\cdot)$, and Borel cohomology for non-equivariantly complex orientable theories.

The purpose of this article is to show that this good behaviour is sufficient to ensure good behaviour of complex vector bundles of any dimension. In particular all complex vector bundles have Thom classes.

Theorem 1.1. If $E$ is complex orientable then any A-equivariant complex vector bundle is E-orientable.

This is proved in Sect. 6.

Okonek [7] has proved that by construction $M U_{A}^{*}(\cdot)$ is universal for cohomology theories with Thom classes. Combined with our main result this implies the following universality statement.

Theorem 1.2. If $E_{A}^{*}(\cdot)$ is a complex oriented cohomology theory with orientation in cohomological degree 2 then there is a unique ring map $M U \longrightarrow E$ of A-spectra under which the orientation of $E$ is the image of the canonical orientation. Conversely, a map $M U \longrightarrow E$ of ring A-spectra endows $E$ with the structure of a complex oriented cohomology theory with orientation in cohomological degree 2.

We include a proof of this in Sect. 7. To make this result somewhat more useful we have the following calculation.

Theorem 1.3. If $E_{A}^{*}(\cdot)$ is a complex orientable theory then $E_{*}^{A}(M U)$ is a polynomial $E_{A}^{*}$-algebra and

$$
\operatorname{Ring}_{A}(M U, E)=E_{*}^{A}-\operatorname{alg}\left(E_{*}^{A}(M U), E_{*}^{A}\right) .
$$

This is proved in Sect. 8. We shall be more specific about the polynomial generators in due course. The good behaviour of $E_{*}^{A}(M U)$ should be contrasted with the facts that $E_{A}^{*}(\mathbb{C} P(\mathcal{U}))$ is not a power series ring in general, and $M U_{A}^{*}$ is not a polynomial algebra.

The main step for all the proofs is the calculation of the cohomology of the universal Grassmannian for any complex oriented cohomology theory. This is also an important step in the calculational exploitation of complex orientable cohomology theories, as is familiar from the non-equivariant case. The traditional methods for the non-equivariant case (see [1] for example) do not apply since the cellular filtrations are not so simple equivariantly. Accordingly, geometric arguments are required as substitutes for the use of the Atiyah-Hirzebruch spectral sequence, and these are illuminating even in the classical case.

We are grateful to the referee for his interesting comments, some of which are reproduced in Remark 5.8. 


\section{Equivariant Grassmannians}

Let $G r_{n}(V)$ be the complex Grassmannian of complex $n$-dimensional subspaces of $V$. For example $G r_{1}(V)=\mathbb{C} P(V)$.

Lemma 2.1. The A-space $G r_{n}(\mathcal{U})$ is a classifying space for A-equivariant complex n-plane bundles:

$$
G r_{n}(\mathcal{U})=B U(n)
$$

We retain the Grassmannian notation, because it will be useful to display the universe explicitly at various points. The direct sum of lines gives a map

$$
\mathbb{C} P(\mathcal{U})^{\times n}=G r_{1}(\mathcal{U})^{\times n} \longrightarrow G r_{n}\left(\mathcal{U}^{\oplus n}\right) \cong G r_{n}(\mathcal{U}),
$$

where the final homeomorphism arises from an isometric isomorphism $\mathcal{U}^{\oplus n} \cong \mathcal{U}$. This sum map induces maps

$$
E_{*}^{A}(\mathbb{C} P(\mathcal{U}))^{\otimes n}=E_{*}^{A}\left(\mathbb{C} P(\mathcal{U})^{\times n}\right) \longrightarrow E_{*}^{A}\left(G r_{n}(\mathcal{U})\right)
$$

and

$$
E_{A}^{*}\left(G r_{n}(\mathcal{U})\right) \longrightarrow E_{A}^{*}\left(\mathbb{C} P(\mathcal{U})^{\times n}\right)=E_{A}^{*}(\mathbb{C} P(\mathcal{U}))^{\hat{\otimes} n},
$$

where the completed tensor producte $\hat{\otimes}$ refers to the skeletal filtration topology. The Künneth theorems implicit in this statement are corollaries of Cole's Splitting Theorem [3].

Since any permutation of the copies of $\mathcal{U}$ in $\mathcal{U}^{\oplus n}$ is homotopic to the identity through isometric isomorphisms, the induced maps factor through coinvariants and invariants for the symmetric group $\Sigma_{n}$.

Theorem 2.2. For any complex orientable cohomology theory $E_{A}^{*}(\cdot)$, the direct sum of lines induces isomorphisms

$$
E_{*}^{A}\left(G r_{n}(\mathcal{U})\right) \cong\left\{E_{*}^{A}(\mathbb{C} P(\mathcal{U}))^{\otimes n}\right\}_{\Sigma_{n}}
$$

and

$$
E_{A}^{*}\left(G r_{n}(\mathcal{U})\right) \cong\left\{E_{A}^{*}(\mathbb{C} P(\mathcal{U}))^{\hat{\otimes} n}\right\}^{\Sigma_{n}} .
$$

Remark 2.3. The homological result can be neatly stated if we take all $n$ together. Indeed the spectrum $\bigvee_{n \geq 0} G r_{n}(\mathcal{U})$ is a ring under direct sum, and

$$
E_{*}^{A}\left(\bigvee_{n \geq 0} G r_{n}(\mathcal{U})\right)=\operatorname{Symm}\left(E_{*}^{A}(\mathbb{C} P(\mathcal{U}))\right) .
$$


Theorem 2.2 will be proved in Sect. 5 below. We pause to remark that this gives us specific generators, and hence all the structure of the homology and cohomology of Grassmannians follows from that of $\mathbb{C} P(\mathcal{U})$ made explicit in [4].

Indeed, Cole showed that $E_{*}^{A}(\mathbb{C} P(\mathcal{U}))$ is additively free over $E_{A}^{*}$ and $E_{A}^{*}(\mathbb{C} P(\mathcal{U}))$ is a product of suspensions of $E_{A}^{*}$. Furthermore, he showed how an orientation $x(\epsilon)$ of $E$, together with a complete flag

$$
\mathcal{F}=\left(0=V^{0} \subset V^{1} \subset V^{2} \subset \cdots\right)
$$

in $\mathcal{U}$ determines a topological basis $1=y\left(V^{0}\right), y\left(V^{1}\right), y\left(V^{2}\right), \ldots$ of $E_{A}^{*}(\mathbb{C} P(\mathcal{U}))$, and we may let $\beta_{0}(\mathcal{F}), \beta_{1}(\mathcal{F}), \beta_{2}(\mathcal{F}), \ldots$ denote the dual basis of $E_{*}^{A}(\mathbb{C} P(\mathcal{U}))$. The notation for the homology generators reflects the fact that $\beta_{i}(\mathcal{F})$ depends on the initial segment $V^{0} \subset V^{1} \subset \cdots \subset V^{i}$ of the flag. Furthermore, a Künneth theorem holds for the homology or cohomology of products of $\mathbb{C} P(\mathcal{U})$.

Lemma 2.4. An $E_{A}^{*}$-basis of the coinvariants $\left\{E_{*}^{A}(\mathbb{C} P(\mathcal{U}))^{\otimes n}\right\}_{\Sigma_{n}}$ is given by the images of all products $\beta_{i_{1}}(\mathcal{F}) \otimes \beta_{i_{2}}(\mathcal{F}) \otimes \cdots \otimes \beta_{i_{n}}(\mathcal{F})$ so that $0 \leq i_{1} \leq i_{2} \leq \cdots \leq i_{n}$. A topological $E_{A}^{*}$-basis of the invariants $\left\{E_{A}^{*}(\mathbb{C} P(\mathcal{U}))^{\hat{\otimes} n}\right\}^{\Sigma_{n}}$ corresponds to the collection of sequences $0 \leq i_{1} \leq$ $i_{2} \leq \cdots \leq i_{n}$; the basis elements are the symmetric sums

$$
\Sigma^{\prime} y\left(V^{i_{\sigma(1)}}\right) \otimes y\left(V^{i_{\sigma(2)}}\right) \otimes \cdots \otimes y\left(V^{i_{\sigma(n)}}\right)
$$

where $\Sigma^{\prime}$ denotes the sum over the orbit of $\left(i_{1}, i_{2}, \ldots, i_{n}\right)$.

Proof. The result is clear once we remark that the $\Sigma_{n}$ action arises from an action on the basis of the homology or cohomology of $\mathbb{C} P(\mathcal{U})^{\times n}$. In the case of cohomology, we pass to limits from the case of $\mathbb{C} P(V)^{\times n}$.

Corollary 2.5. If $E$ is a complex oriented cohomology theory then $E \wedge$ $G r_{n}(\mathcal{U})$ splits as a wedge of copies of $E$ indexed by sequences $0 \leq i_{1} \leq$ $i_{2} \leq \cdots \leq i_{n}$ :

$$
E \wedge G r_{n}(\mathcal{U}) \simeq \bigvee_{\mathbf{i}} \Sigma^{2|\mathbf{i}|} E,
$$

where $|\mathbf{i}|=i_{1}+i_{2}+\cdots+i_{n}$. The splitting depends on the orientation.

Proof. In the usual way, from the basis of $E_{*}^{A}\left(G r_{n}(\mathcal{U})\right)$, we may construct a map

$$
s: \bigvee_{\mathbf{i}} \Sigma^{2|\mathbf{i}|} E \longrightarrow E \wedge G r_{n}(\mathcal{U})
$$

of $A$-spectra, using the product on $E$.

By construction it induces an isomorphism in $\pi_{*}^{A}(\cdot)$. Now suppose $B \subseteq$ $A$, and consider the $B$-equivariant situation. The restriction map $E_{A}^{*}(\mathbb{C} P(\mathcal{U}))$ 
$\longrightarrow E_{B}^{*}(\mathbb{C} P(\mathcal{U}))$ takes an $A$-orientation to a $B$-orientation, and the resulting basis corresponding to a complete flag to the basis corresponding to the same flag regarded $B$-equivariantly. It therefore follows that the restriction $E_{A}^{*}\left(G r_{n}(\mathcal{U})\right) \longrightarrow E_{B}^{*}\left(G r_{n}(\mathcal{U})\right)$ takes the sequence basis to another basis. Thus $s$ induces an isomorphism of $\pi_{*}^{B}(\cdot)$. Since this applies to all subgroups $B$ of $A$, the map $s$ is an $A$-equivalence by the Whitehead theorem.

It may be useful to record the calculation relative to a specific orientation in very concrete terms.

Corollary 2.6. If E is a complex oriented cohomology theory then

$$
\begin{aligned}
E_{*}^{A}\left(G r_{n}(\mathcal{U})\right) & =E_{*}^{A}\left\{\beta\left(V^{i_{1}}\right) \otimes \beta\left(V^{i_{2}}\right)\right. \\
& \left.\otimes \cdots \otimes \beta\left(V^{i_{n}}\right) \mid 0 \leq i_{1} \leq i_{2} \leq \cdots \leq i_{n}\right\}
\end{aligned}
$$

and

$$
\begin{aligned}
E_{A}^{*}\left(G r_{n}(\mathcal{U})\right) & =E_{*}^{A}-\bmod \left(E_{*}^{A}\left(G r_{n}(\mathcal{U})\right), E_{*}^{A}\right) \\
& =E_{A}^{*}\left\{\left\{\Sigma^{\prime} y\left(V^{i_{\sigma(1)}}\right) \otimes y\left(V^{i_{\sigma(2)}}\right)\right.\right. \\
& \left.\left.\otimes \cdots \otimes y\left(V^{i_{\sigma(n)}}\right) \mid 0 \leq i_{1} \leq i_{2} \leq \cdots \leq i_{n}\right\}\right\}
\end{aligned}
$$

Finally we may consider the extra structure on the system

$$
\left\{E_{*}^{A}\left(G r_{n}(\mathcal{U})\right)\right\}_{n \geq 0} \text { or }\left\{E_{A}^{*}\left(G r_{n}(\mathcal{U})\right)\right\}_{n \geq 0} .
$$

The relevant extra structure is given by the conjugation map

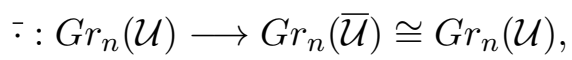

the action maps

$$
\otimes \alpha: G r_{n}(\mathcal{U}) \longrightarrow G r_{n}(\mathcal{U} \otimes \alpha) \cong G r_{n}(\mathcal{U})
$$

for $\alpha \in A^{*}$, the direct sum maps

$$
\oplus: G r_{m}(\mathcal{U}) \times G r_{n}(\mathcal{U}) \longrightarrow G r_{m+n}(\mathcal{U} \oplus \mathcal{U}) \cong G r_{m+n}(\mathcal{U})
$$

and the tensor product maps

$$
\otimes: G r_{m}(\mathcal{U}) \times G r_{n}(\mathcal{U}) \longrightarrow G r_{m n}(\mathcal{U} \otimes \mathcal{U}) \cong G r_{m n}(\mathcal{U})
$$

The structure constants for all these maps in homology and cohomology can be deduced from those $\otimes \alpha$ for $n=1$ and $\otimes$ for $m=n=1$. Furthermore the coproduct on $E_{*}^{A}\left(G r_{n}(\mathcal{U})\right)$ and the product on $E_{A}^{*}\left(G r_{n}(\mathcal{U})\right)$ may be deduced from the corresponding structure for $\mathbb{C} P(\mathcal{U})$. These facts, like all our methods, depend crucially on the fact that the group $A$ is abelian, so that all representations are sums of one dimensional representations. 


\section{The equivariant Schubert cells of a Grassmannian}

Consider the decomposition of $G r_{n}(V)$ into Schubert cells, as described for example in [6]. We choose a complete $A$-invariant flag

$$
0=V^{0} \subset V^{1} \subset V^{2} \subset V^{3} \subset \cdots \subset V^{m}=V,
$$

and let $\alpha_{i}=V^{i} / V^{i-1}$ as usual. It is well known that $G r_{n}(V)$ admits a non-equivariant $\mathrm{CW}$-structure in which the cells are indexed by sequences of integers

$$
1 \leq \sigma_{1}<\sigma_{2}<\cdots<\sigma_{n} \leq m ;
$$

the sequence $\sigma=\left(\sigma_{1}, \sigma_{2}, \ldots, \sigma_{n}\right)$ is called a Schubert symbol. It is convenient to use $\sigma$ to select a complete flag

$$
0=V(\sigma)^{0} \subset V(\sigma)^{1} \subset V(\sigma)^{2} \subset V(\sigma)^{3} \subset \cdots \subset V(\sigma)^{n}
$$

of length $n$ where

$$
V(\sigma)^{i}=\alpha_{\sigma_{1}} \oplus \alpha_{\sigma_{2}} \oplus \cdots \oplus \alpha_{\sigma_{i}}
$$

The cell $e(\sigma)$ corresponding to the Schubert symbol $\sigma$ consists of all $n$ planes $X$ with

$$
\operatorname{dim}\left(X \cap V^{\sigma_{i}}\right)=i \text { and } \operatorname{dim}\left(X \cap V^{\sigma_{i}-1}\right)=i-1
$$

for $i=1,2, \ldots, n$. Such an $n$-plane $X$ admits a basis $x_{1}, x_{2}, \ldots, x_{n}$ with $x_{i} \in V^{\sigma_{i}}$ and non-zero in $V^{\sigma_{i}} / V^{\sigma_{i}-1}$. This is usually represented as an $m \times$ $n$-matrix with rows $x_{1}, x_{2}, \ldots, x_{n}$, and columns indexed by $\alpha_{1}, \alpha_{2}, \ldots, \alpha_{m}$. Dividing $x_{i}$ by its $\alpha_{\sigma_{i}}$ coordinate, we may assume the last entry in each row is 1 , and then subtracting a suitable multiple of $x_{i}$ from the other rows we may assume the matrix is in row-reduced echelon form.

Note that since $V^{j}$ is $A$-invariant, the cell $e(\sigma)$ is an $A$-subspace. Accordingly, these same Schubert cells $e(\sigma)$ give a decomposition as an equivariant $\operatorname{Rep}(A)-\mathrm{CW}$-complex, in the sense that the cells are unit discs in complex representations of $A$, and attached to cells corresponding to proper summands. Indeed, the $i$ th row gives the representation $\alpha_{\sigma_{i}}^{-1} \otimes\left(V^{\sigma_{i}} / V(\sigma)^{i}\right)$, so that the cell $e(\sigma)$ is of $\operatorname{Rep}(A)$-dimension

$$
\bigoplus_{i=1}^{n} \alpha_{\sigma_{i}}^{-1} \otimes\left(V^{\sigma_{i}} / V(\sigma)^{i}\right) .
$$




\section{Thom complexes and the Schubert filtration}

There is a convenient filtration associated to the Schubert cells, which we shall need to use. We let $G r_{n}(\mathcal{U})^{[k]}$ be the subcomplex of $G r_{n}(\mathcal{U})$ corresponding to Schubert cells with $\sigma_{1} \leq k+1$; the indexing is chosen since $k+1$ is the lowest complex dimension of a cell not in $G r_{n}(\mathcal{U})^{[k]}$. The resulting filtration

$$
G r_{n}(\mathcal{U})^{[0]} \subset G r_{n}(\mathcal{U})^{[1]} \subset G r_{n}(\mathcal{U})^{[2]} \subset \cdots \subset G r_{n}(\mathcal{U})
$$

will be called the Schubert filtration. Of course the Schubert filtration depends on the complete flag $\mathcal{F}$; when we use the universe $\mathcal{U}-V^{k}$, we use the cell structure associated to the complete flag $\mathcal{F} / V^{k}$.

To obtain sufficient naturality we need to interpret the filtration in terms of Thom complexes. For this we let $\gamma_{n}$ denote the tautological $n$-plane bundle over $G r_{n}(\mathcal{U})$ as usual.

The dimensions of the $\operatorname{Rep}(A)$-cells suggest the plausibility of the following result.

Theorem 4.1. There is a homotopy equivalence

$$
G r_{n}(\mathcal{U}) / G r_{n}(\mathcal{U})^{[k-1]} \simeq\left(G r_{n}\left(\mathcal{U}-V^{k}\right)\right)^{\operatorname{Hom}\left(\gamma_{n}, V^{k}\right)} .
$$

This may be chosen natural as $k$ varies in the sense that there is a homotopy commutative diagram

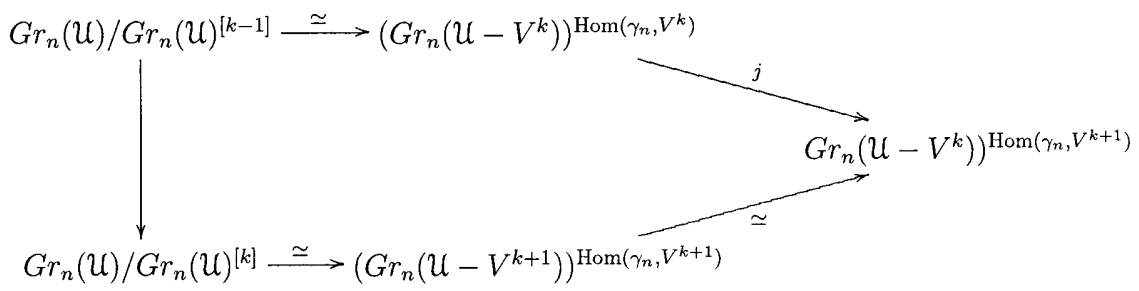

where the left hand vertical is the quotient map, and $j$ is induced by the inclusion $V^{k} \subseteq V^{k+1}$.

Remark 4.2. Since $\mathcal{U}$ is a complete $A$-universe, for any finite dimensional subspace $V \subset \mathcal{U}$, the inclusion $\mathcal{U}-V \subseteq \mathcal{U}$ induces the stabilization equivalence

$$
G r_{n}(\mathcal{U}-V) \simeq G r_{n}(\mathcal{U}) .
$$

Proof. The proof is by induction on $k$, using the following two equivalences.

Lemma 4.3. There is an equvialence

$$
G r_{n}\left(\mathcal{U}-V^{k}\right) / G r_{n}\left(\mathcal{U}-V^{k}\right)^{[0]} \simeq G r_{n}\left(\mathcal{U}-V^{k+1}\right)^{\operatorname{Hom}\left(\gamma_{n}, \alpha_{k+1}\right)},
$$


Remark 4.4. In view of the fact that $G r_{n}\left(\mathcal{U}-V^{k}\right)^{[0]} \cong G r_{n-1}\left(\mathcal{U}-V^{k+1}\right)$ the lemma may be viewed as giving a Gysin cofibre sequence

$$
\begin{aligned}
G r_{n-1}\left(\mathcal{U}-V^{k+1}\right) & \stackrel{\alpha_{k+1} \oplus}{\longrightarrow} G r_{n}\left(\mathcal{U}-V^{k}\right) \\
& \longrightarrow G r_{n}\left(\mathcal{U}-V^{k+1}\right)^{\operatorname{Hom}\left(\gamma_{n}, \alpha_{k+1}\right)}
\end{aligned}
$$

Lemma 4.5. There is a homeomorphism

$$
G r_{n}(\mathcal{U})^{[k]} / G r_{n}(\mathcal{U})^{[k-1]} \cong\left(G r_{n}\left(\mathcal{U}-V^{k}\right)^{[0]}\right)^{\operatorname{Hom}\left(\gamma_{n}, V^{k}\right)} .
$$

Using these we may complete the proof. First note that taking $k=0$ in 4.3 gives the base of the induction. Now suppose that $G r_{n}(\mathcal{U}) / G r_{n}(\mathcal{U})^{[k-1]}$ has been identified as in the statement of the theorem. We then calculate

$$
\begin{aligned}
G r_{n}(\mathcal{U}) / G r_{n}(\mathcal{U})^{[k]} & =\left(G r_{n}(\mathcal{U}) / G r_{n}(\mathcal{U})^{[k-1]}\right) /\left(G r_{n}(\mathcal{U})^{[k]} / G r_{n}(\mathcal{U})^{[k-1]}\right) \\
& \simeq\left\{G r_{n}\left(\mathcal{U}-V^{k}\right) / G r_{n}\left(\mathcal{U}-V^{k}\right)^{[0]}\right\}^{\operatorname{Hom}\left(\gamma_{n}, V^{k}\right)} \\
& \simeq\left\{G r_{n}\left(\mathcal{U}-V^{k+1}\right)^{\operatorname{Hom}\left(\gamma_{n}, \alpha_{k+1}\right)}\right\}^{\operatorname{Hom}\left(\gamma_{n}, V^{k}\right)} \\
& \simeq G r_{n}\left(\mathcal{U}-V^{k+1}\right)^{\operatorname{Hom}\left(\gamma_{n}, V^{k+1}\right)},
\end{aligned}
$$

where the first equivalence uses the inductive hypothesis and 4.5, and the second uses 4.3.

It therefore remains to prove the two lemmas.

Proof of 4.3. We view the Thom space as the mapping cone of the projection of the unit sphere bundle. It therefore suffices to construct a homotopy commutative square

$$
\begin{aligned}
& S\left(\operatorname{Hom}\left(\gamma_{n}, \alpha_{k+1}\right)\right) \longrightarrow G r_{n}\left(\mathcal{U}-V^{k+1}\right)
\end{aligned}
$$

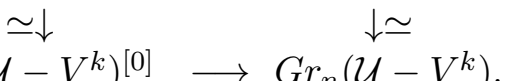

$$
\begin{aligned}
& G r_{n}\left(\mathcal{U}-V^{k}\right)^{[0]} \longrightarrow G r_{n}\left(\mathcal{U}-V^{k}\right) .
\end{aligned}
$$

The right hand equivalence is the stabilization equivalence induced by the inclusion of complete universes. The right hand vertical $S\left(\operatorname{Hom}\left(\gamma_{n}, \alpha_{k+1}\right)\right)$ $\longrightarrow G r_{n}\left(\mathcal{U}-V^{k}\right)^{[0]}$ is defined by $(z, X) \longmapsto \alpha_{k+1} \oplus \operatorname{ker}(z)$, where $z$ is a unit vector in $\operatorname{Hom}\left(X, \alpha_{k+1}\right)$. To see it is an equivalence, we note that the fibre over $Y$ is the unit sphere in $\mathcal{U}-\left(V^{k} \oplus Y\right)$.

To see the square commutes up to homotopy, note that the two routes round the square can both be interpreted as sending $(z, X)$ to the kernel of a suitable map $f: \alpha_{k+1} \oplus X \longrightarrow \alpha_{k+1}$. For the lower route $f(w, x)=$ $z(x)$, and for the upper route $f(w, x)=w$. The interval joining these two maps consists of nonzero linear maps and therefore provides a homotopy as required. 
Proof of 4.5. For this it is convenient to view the Thom space as a compactification of the vector bundle. We may then define a map

$$
G r_{n}(\mathcal{U})^{[k]} / G r_{n}(\mathcal{U})^{[k-1]} \longrightarrow\left(G r_{n}\left(\mathcal{U}-V^{k}\right)^{[0]}\right)^{\operatorname{Hom}\left(\gamma_{n}, V^{k}\right)}
$$

by taking a representative $n$-plane $X$, expressed in standard form as $X=$ $\left(X_{\leq k} \mid X_{>k}\right)$, to the vector $X_{\leq k}$ in the fibre over $X_{>k}$. This is clearly a homeomorphism away from the basepoint, and one may check it is equivariant and that both it and its inverse are continuous at the basepoint.

This completes the proof of 4.1 .

\section{The spectral sequence of universal Thom complexes}

We now construct a spectral sequence for calculating $E_{*}^{A}\left(G r_{n}(\mathcal{U})\right)$ using the Schubert cell filtration

$$
G r_{n}(\mathcal{U})^{[0]} \subset G r_{n}(\mathcal{U})^{[1]} \subset G r_{n}(\mathcal{U})^{[2]} \subset \cdots \subset G r_{n}(\mathcal{U})
$$

introduced in Sect. 4. We think of the superscript $k$ in $G r_{n}(\mathcal{U})^{[k]}$ as a complex dimension, and applying $E_{*}^{A}(\cdot)$ to the filtration we obtain a right half-plane homological spectral sequence $E(1)_{*, *}^{*}$ concentrated in even filtration degrees with

$$
E(1)_{2 p, q}^{1}=E_{2 p+q}^{A}\left(G r_{n}(\mathcal{U})^{[p]}, G r_{n}(\mathcal{U})^{[p-1]}\right) \Longrightarrow E_{2 p+q}^{A}\left(G r_{n}(\mathcal{U})\right) .
$$

The spectral sequence is indexed so that a $\operatorname{Rep}(A)-s$-cell in filtration $2 p$ contributes to $E_{2 p, 2 s}^{1}$. There is an analogous spectral sequence for cohomology obtained by applying $E_{A}^{*}(\cdot)$ to the filtration. It is a right half-plane cohomological spectral sequence concentrated in even filtration degrees:

$$
E(1)_{1}^{2 p, q}=E_{A}^{2 p+q}\left(G r_{n}(\mathcal{U})^{[p]}, G r_{n}(\mathcal{U})^{[p-1]}\right) \Longrightarrow E_{A}^{2 p+q}\left(G r_{n}(\mathcal{U})\right) .
$$

Remark 5.1. In view of the equivalence

$$
G r_{n}(\mathcal{U}) / G r_{n}(\mathcal{U})^{[k-1]} \simeq G r_{n}\left(\mathcal{U}-V^{k}\right)^{\operatorname{Hom}\left(\gamma_{n}, V^{k}\right)}
$$

obtained from 4.1 by stabilization, the same spectral sequences may be obtained from the sequence of maps

$$
\begin{aligned}
G r_{n}(\mathcal{U})^{0} & \longrightarrow G r_{n}(\mathcal{U})^{\operatorname{Hom}\left(\gamma_{n}, V^{1}\right)} \longrightarrow G r_{n}(\mathcal{U})^{\operatorname{Hom}\left(\gamma_{n}, V^{2}\right)} \\
& \longrightarrow G r_{n}(\mathcal{U})^{\operatorname{Hom}\left(\gamma_{n}, V^{3}\right)} \longrightarrow \ldots
\end{aligned}
$$

This point of view is useful for establishing naturality. 
Proposition 5.2. If $E$ is a complex oriented cohomology theory then the homological spectral sequence $E(1)_{*, *}^{*}$ collapses for all n. In fact $E_{*}^{A}\left(G r_{n}(\mathcal{U})\right)$ is free over $E_{A}^{*}$ on the basis of all products $\beta_{i_{1}}(\mathcal{F}) \otimes \beta_{i_{2}}(\mathcal{F}) \otimes \cdots \otimes \beta_{i_{n}}(\mathcal{F})$ so that $0 \leq i_{1} \leq i_{2} \leq \cdots \leq i_{n}$ as described in 2.4 .

Proof. We prove 2.2 and 5.2 by simultaneous induction on $n$. The case $n=1$ is given by Cole's Splitting Theorem [3], so we may suppose $n \geq 2$ and that the results have been proved for all smaller values. In particular we have Thom isomorphisms for all vector bundles of dimension $\leq n-1$ (see Sect. 6 for more details).

We consider the embedding

$$
\mathbb{C} P(\mathcal{U}) \times G r_{n-1}(\mathcal{U})=G r_{1}(\mathcal{U}) \times G r_{n-1}(\mathcal{U}) \stackrel{\oplus}{\longrightarrow} G r_{n}(\mathcal{U} \oplus \mathcal{U}) \cong G r_{n}(\mathcal{U}) .
$$

The proof proceeds by constructing a compatible spectral sequence $E(2)_{*, *}^{*}$ for calculating $E_{*}^{A}\left(\mathbb{C} P(\mathcal{U}) \times G r_{n-1}(\mathcal{U})\right)$. By induction the new spectral sequence $E(2)_{*, *}^{*}$ calculates known groups, and will be shown to collapse. On the other hand the map $E(2)_{*, *}^{*} \longrightarrow E(1)_{*, *}^{*}$ of spectral sequences will be shown to be surjective on $E^{1}$-terms, so it follows that $E(1)_{*, *}^{*}$ collapses as required.

We proceed to construct the new spectral sequence. The analogue of 4.4 is as follows.

Lemma 5.3. There is a cofibre sequence

$$
\begin{aligned}
X(k)_{+} & \longrightarrow\left(\mathbb{C} P\left(\mathcal{U}-V^{k}\right) \times G r_{n-1}\left(\mathcal{U}-V^{k}\right)\right)_{+} \\
& \longrightarrow\left(\mathbb{C} P\left(\mathcal{U}-V^{k+1}\right) \times G r_{n-1}\left(\mathcal{U}-V^{k+1}\right)\right)^{\operatorname{Hom}\left(\gamma_{n}, \alpha_{k+1}\right)}
\end{aligned}
$$

where $X(k)$ is a pushout

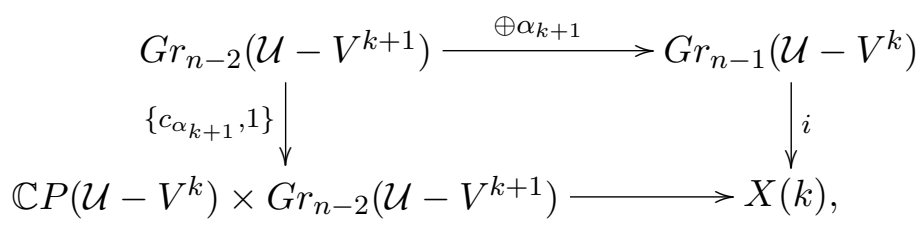

where the top horizontal is induced by adding $\alpha_{k+1}$ to the $(n-1)$-plane and universe as in 4.4, and the left hand vertical uses the inclusion of the $A$-fixed line $\alpha_{k+1}$ in $\mathbb{C P}\left(\mathcal{U}-V^{k}\right)$ in the first factor.

Proof. Over $\mathbb{C} P\left(\mathcal{U}-V^{k+1}\right) \times G r_{n-1}\left(\mathcal{U}-V^{k+1}\right)$ the bundle $\operatorname{Hom}\left(\gamma_{n}, \alpha_{k+1}\right)$ is the product $\operatorname{Hom}\left(\gamma_{1}, \alpha_{k+1}\right) \times \operatorname{Hom}\left(\gamma_{n-1}, \alpha_{k+1}\right)$. Thus the unit disc bundle is the product $D\left(\operatorname{Hom}\left(\gamma_{1}, \alpha_{k+1}\right)\right) \times D\left(\operatorname{Hom}\left(\gamma_{n-1}, \alpha_{k+1}\right)\right)$ and the Thom space is the smash product

$$
\mathbb{C} P\left(\mathcal{U}-V^{k+1}\right)^{\operatorname{Hom}\left(\gamma_{1}, \alpha_{k+1}\right)} \wedge G r_{n-1}\left(\mathcal{U}-V^{k+1}\right)^{\operatorname{Hom}\left(\gamma_{n-1}, \alpha_{k+1}\right)} .
$$


This identifies the unit sphere bundle $S\left(\operatorname{Hom}\left(\gamma_{1}, \alpha_{k+1}\right) \times \operatorname{Hom}\left(\gamma_{n-1}, \alpha_{k+1}\right)\right)$ as the pushout

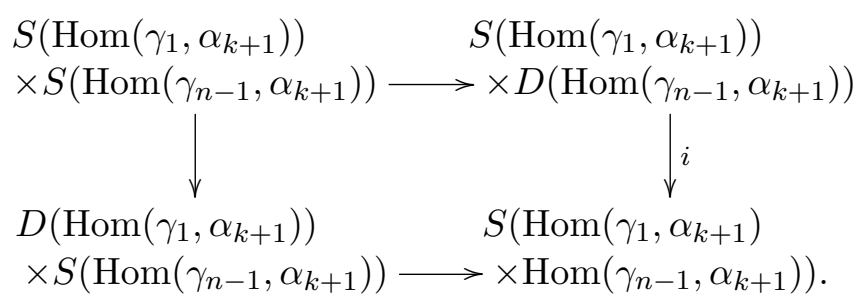

The description of $X(k)$ follows.

Corollary 5.4. There is a cofibre sequence

$$
\begin{aligned}
X(k)^{\operatorname{Hom}\left(\gamma_{n}, V^{k}\right)} & \longrightarrow\left(\mathbb{C} P\left(\mathcal{U}-V^{k}\right) \times G r_{n-1}\left(\mathcal{U}-V^{k}\right)\right)^{\operatorname{Hom}\left(\gamma_{n}, V^{k}\right)} \\
& \longrightarrow\left(\mathbb{C} P\left(\mathcal{U}-V^{k+1}\right) \times G r_{n-1}\left(\mathcal{U}-V^{k+1}\right)^{\operatorname{Hom}\left(\gamma_{n}, V^{k+1}\right)} .\right.
\end{aligned}
$$

In view of the inductive hypothesis we know the homologies of all the spaces involved in the cofibre sequence of 5.3, and we have a basis corresponding to a $\operatorname{Rep}(A)-\mathrm{CW}$-decomposition (where the correspondence depends on the orientation). We return to analysis of the maps and the bases below.

Corollary 5.5. The map

$$
\mathbb{C} P\left(\mathcal{U}-V^{k+1}\right) \times G r_{n-1}\left(\mathcal{U}-V^{k+1}\right) \stackrel{\oplus}{\longrightarrow} G r_{n}\left(\mathcal{U}-V^{k+1}\right)
$$

induces a retraction

$$
\begin{aligned}
r: X(k) & =S\left(\operatorname{Hom}\left(\gamma_{1}, \alpha_{k+1}\right) \times \operatorname{Hom}\left(\gamma_{n-1}, \alpha_{k+1}\right)\right) \\
& \longrightarrow S\left(\operatorname{Hom}\left(\gamma_{n}, \alpha_{k+1}\right)\right) \simeq G r_{n-1}\left(\mathcal{U}-V^{k}\right)
\end{aligned}
$$

on unit sphere bundles.

Proof. The inclusion corresponding to the retraction $r$ is the composite

$$
\begin{aligned}
& G r_{n-1}\left(\mathcal{U}-V^{k}\right) \simeq S\left(\operatorname{Hom}\left(\gamma_{1}, \alpha_{k+1}\right)\right) \times D\left(\operatorname{Hom}\left(\gamma_{n-1}, \alpha_{k+1}\right)\right) \stackrel{i}{\longrightarrow} \\
& S\left(\operatorname{Hom}\left(\gamma_{1}, \alpha_{k+1}\right) \times \operatorname{Hom}\left(\gamma_{n-1}, \alpha_{k+1}\right)\right)=X(k) .
\end{aligned}
$$

It is easily checked that with the equivalence $S\left(\operatorname{Hom}\left(\gamma_{n}, \alpha_{k+1}\right)\right) \simeq$ $G r_{n-1}\left(\mathcal{U}-V^{k}\right)$ specified in 4.3, we have $r i=1$. 
To guarantee a map of spectral sequences $E(2)_{*, *}^{*} \longrightarrow E(1)_{*, *}^{*}$ converging to the map

$$
E_{*}^{A}\left(\mathbb{C} P(\mathcal{U}) \times G r_{n-1}(\mathcal{U})\right) \longrightarrow E_{*}^{A}\left(G r_{n}(\mathcal{U})\right),
$$

the spectral sequence for $E_{*}^{A}\left(\mathbb{C} P(\mathcal{U}) \times G r_{n-1}(\mathcal{U})\right)$ is constructed from the sequence of maps

$$
\begin{aligned}
\left(\mathbb{C} P(\mathcal{U}) \times G r_{n-1}(\mathcal{U})\right)^{0} & \longrightarrow\left(\mathbb{C} P(\mathcal{U}) \times G r_{n-1}(\mathcal{U})\right)^{\operatorname{Hom}\left(\gamma_{n}, V^{1}\right)} \\
& \longrightarrow\left(\mathbb{C} P(\mathcal{U}) \times G r_{n-1}(\mathcal{U})\right)^{\operatorname{Hom}\left(\gamma_{n}, V^{2}\right)} \longrightarrow \cdots
\end{aligned}
$$

as in 5.1. However, it may help if we relate this to a construction more closely analogous to the construction of $E(1)_{*, *}^{*}$ from the Schubert filtration. Note that over $\mathbb{C} P(\mathcal{U}) \times G r_{n-1}(\mathcal{U})$ the bundle $\operatorname{Hom}\left(\gamma_{n}, V^{k}\right)$ is $\operatorname{Hom}\left(\gamma_{1}, V^{k}\right) \times$ $\operatorname{Hom}\left(\gamma_{n-1}, V^{k}\right)$, and hence

$$
\begin{aligned}
& \left(\mathbb{C} P(\mathcal{U}) \times G r_{n-1}(\mathcal{U})\right)^{\operatorname{Hom}\left(\gamma_{n}, V^{k}\right)} \\
& \simeq \mathbb{C} P(\mathcal{U})^{\operatorname{Hom}\left(\gamma_{1}, V^{k}\right)} \wedge G r_{n-1}(\mathcal{U})^{\operatorname{Hom}\left(\gamma_{n-1}, V^{k}\right)} .
\end{aligned}
$$

We therefore define

$$
\begin{aligned}
\left(\mathbb{C} P(\mathcal{U}) \times G r_{n-1}(\mathcal{U})\right)^{[k]}:= & \mathbb{C} P(\mathcal{U}) \times\left(G r_{n-1}(\mathcal{U})^{[k]}\right) \cup \\
& \left(\mathbb{C} P(\mathcal{U})^{[k]}\right) \times G r_{n-1}(\mathcal{U}),
\end{aligned}
$$

so as to ensure a cofibre sequence

$$
\begin{aligned}
\left(\mathbb{C} P(\mathcal{U}) \times G r_{n-1}(\mathcal{U})\right)^{[k-1]} \longrightarrow \mathbb{C} P(\mathcal{U}) \times G r_{n-1}(\mathcal{U}) \longrightarrow \\
\left(\mathbb{C} P\left(\mathcal{U}-V^{k}\right) \times G r_{n-1}\left(\mathcal{U}-V^{k}\right)\right)^{\operatorname{Hom}\left(\gamma_{n}, V^{k}\right)} .
\end{aligned}
$$

Applying $E_{*}^{A}(\cdot)$ to the resulting filtration we obtain a right half-plane homological spectral sequence with

$$
\begin{aligned}
E(2)_{2 p, q}^{1} & =E_{2 p+q}^{A}\left(\left(\mathbb{C} P(\mathcal{U}) \times G r_{n-1}(\mathcal{U})\right)^{[p]},\left(\mathbb{C} P(\mathcal{U}) \times G r_{n-1}(\mathcal{U})\right)^{[p-1]}\right) \\
& \Longrightarrow E_{2 p+q}^{A}\left(\mathbb{C} P(\mathcal{U}) \times G r_{n-1}(\mathcal{U})\right) .
\end{aligned}
$$

By induction this spectral sequence is under complete control.

Proposition 5.6. If $E_{A}^{*}(\cdot)$ is a complex oriented cohomology theory then the spectral sequence $E(2)_{*, *}^{*}$ above collapses at $E(2)_{*, *}^{1}$.

Proof. This follows from the inductive hypothesis, since $E \wedge G r_{n-1}(\mathcal{U})_{+}$ splits as a wedge of suspensions of $E$ indexed by the Schubert cells of $G r_{n-1}(\mathcal{U})_{+}$. Smashing this with $\mathbb{C} P(\mathcal{U})_{+}$, we again get splitting from Cole's Splitting Theorem [3]. The filtration of the new spectral sequence is compatible with that of the splitting in view of the smash product decomposition of the Thom spaces. The collapse of the spectral sequence follows. 
To see that the map $E(2)_{1}^{*, *} \longrightarrow E(1)_{1}^{*, *}$ is surjective, recall that

$$
G r_{n}(\mathcal{U})^{[k]} / G r_{n}(\mathcal{U})^{[k-1]} \simeq G r_{n-1}\left(\mathcal{U}-V^{k}\right)^{\operatorname{Hom}\left(\gamma_{n}, V^{k}\right)}
$$

and

$$
\begin{aligned}
\left(\mathbb{C} P(\mathcal{U}) \times G r_{n-1}(\mathcal{U})\right)^{[k]} /\left(\mathbb{C} P\left(\mathcal{U}-V^{k}\right)\right. & \left.\times G r_{n-1}\left(\mathcal{U}-V^{k}\right)\right)^{[k-1]} \\
& \simeq X(k)^{\operatorname{Hom}\left(\gamma_{n}, V^{k}\right)} .
\end{aligned}
$$

It therefore suffices to show that for each $k$, the map

$$
X(k)^{\operatorname{Hom}\left(\gamma_{n}, V^{k}\right)} \longrightarrow G r_{n-1}\left(\mathcal{U}-V^{k}\right)^{\operatorname{Hom}\left(\gamma_{n}, V^{k}\right)}
$$

of subquotients is surjective in $E_{*}^{A}(\cdot)$. This follows for the unThomified map, since by 5.5 the map $r: X(k) \longrightarrow G r_{n-1}\left(\mathcal{U}-V^{k}\right)$ is a retraction. For $k=0$ this is the required statement. For $k \geq 1$ we use the fact that over $\mathbb{C} P\left(\mathcal{U}-V^{k}\right) \times G r_{n-1}\left(\mathcal{U}-V^{k}\right)$ the bundle $\gamma_{n}$ splits as the product $\gamma_{1} \times \gamma_{n-1}$. Thus $\operatorname{Hom}\left(\gamma_{n}, V^{k}\right)$ splits as a sum of bundles of dimension $\leq n-1$ for each of which we have a Thom isomorphism by induction. Surjectivity now follows from that of the unThomified map for the universe $\mathcal{U}-V^{k}$.

Finally, we may remark that the analysis identifies a basis. We may visualize the basis of the homology $\mathbb{C} P(\mathcal{U})^{\times n}$ as the points of $\mathbb{N}^{n}$, with the complex dimension of the cell corresponding to $\mathbf{i}=\left(i_{1}, i_{2}, \ldots, i_{n}\right)$ being $|\mathbf{i}|=i_{1}+i_{2}+\cdots+i_{n}$. The homology of all other relevant spaces have bases given by the images of these basis elements. These correspond to subsets of $\mathbb{N}^{n}$ as follows. The basis for the space $\mathbb{C} P(\mathcal{U})=\mathbb{C} P(\mathcal{U}) \times *$ corresponds to the points $\left(i_{1}, 0, \ldots, 0\right)$, and that for $G r_{n-1}(\mathcal{U})=* \times$ $G r_{n-1}(\mathcal{U})$ corresponds to the points $\left(0, i_{2}, \ldots, i_{n}\right)$ with $0 \leq i_{2} \leq i_{3} \leq$ $\cdots \leq i_{n}$. The basis for the subspace $G r_{n-2}(\mathcal{U}) \subseteq G r_{n-1}(\mathcal{U})$ corresponds to the points $\left(0,0, i_{3}, \ldots, i_{n}\right)$ with $0 \leq i_{3} \leq i_{4} \leq \cdots \leq i_{n}$.

Applying this discussion to the universe $\mathcal{U}-V^{k}$, we see that $X(k)$ has homology in even degrees, with basis corresponding to the points $\left(i_{1}, i_{2}, \ldots, i_{n}\right)$ either (i) with $i_{1}=0$ and $0 \leq i_{2} \leq i_{3} \leq \cdots \leq i_{n}$ or (ii) with $i_{1}$ arbitrary, $i_{2}=0$ and $0 \leq i_{3} \leq i_{4} \leq \cdots \leq i_{n}$. Since the map $i$ occurs in the pushout description of $X(k)$ we see that $r: X(k) \longrightarrow G r_{n-1}\left(\mathcal{U}-V^{k}\right)$ maps the subspace on the basis elements elements corresponding to $0 \leq i_{2} \leq i_{3} \leq$ $\cdots \leq i_{n}$ isomorphically to the homology of $G r_{n-1}\left(\mathcal{U}-V^{k}\right)$. The effect of the Thom isomorphism is to replace standard homology generators relative to the flag $\mathcal{F} / V^{k}$ by those for $\mathcal{F}$ : the precise meaning becomes clear from the special case $\mathbb{C} P(\mathcal{U})$.

Lemma 5.7. Naming the cohomology $E_{A}^{*}\left(\mathbb{C} P\left(\mathcal{U}-V^{k}\right)^{\operatorname{Hom}\left(\gamma_{1}, V^{k}\right)}\right)$ by the equivalence $\mathbb{C} P\left(\mathcal{U}-V^{k}\right)^{\operatorname{Hom}\left(\gamma_{1}, V^{k}\right)} \simeq \mathbb{C} P(\mathcal{U}) / \mathbb{C} P\left(V^{k}\right)$, the element 
$y\left(V^{k}\right)$ is a Thom class. The resulting Thom isomorphisms $E_{A}^{*}(\mathbb{C} P(\mathcal{U}-$ $\left.\left.V^{k}\right)^{\operatorname{Hom}\left(\gamma_{1}, V^{k}\right)}\right) \cong E_{A}^{*}\left(\mathbb{C} P\left(\mathcal{U}-V^{k}\right)\right.$ and $E_{*}^{A}\left(\mathbb{C} P\left(\mathcal{U}-V^{k}\right)^{\operatorname{Hom}\left(\gamma_{1}, V^{k}\right)}\right) \cong$ $E_{*}^{A}\left(\mathbb{C} P\left(\mathcal{U}-V^{k}\right)\right.$ are given by

$$
y\left(V^{k+l} / V^{k}\right) \longmapsto y\left(V^{k}\right) y\left(V^{k+l} / V^{k}\right)=y\left(V^{k+l}\right)
$$

in cohomology and

$$
\beta_{l}\left(\mathcal{F} / V^{k}\right) \longleftarrow \beta_{k+l}(\mathcal{F})
$$

in homology for $l \geq 0$.

Now we return to find a basis for $E_{*}^{A}\left(G r_{n}(\mathcal{U})\right)$ itself. By induction we know that the elements $\beta_{i_{1}}(\mathcal{F}) \otimes \beta_{i_{2}}(\mathcal{F}) \otimes \beta_{i_{3}}(\mathcal{F}) \otimes \cdots \otimes \beta_{i_{n}}(\mathcal{F})$ with $i_{1}$ arbitrary and $0 \leq i_{2} \leq i_{3} \leq \cdots \leq i_{n}$ give a basis of $E_{*}^{A}(\mathbb{C} P(\mathcal{U}) \times$ $\left.G r_{n-1}(\mathcal{U})\right)$. The proof of this shows that the filtration is such that the basis of $E(2)_{2 p, *}^{1}$ is given by the images of the elements either

$(i)_{p}$ with $p=i_{1} \leq i_{2} \leq i_{3} \leq \cdots \leq i_{n}$ or

$(\text { (ii })_{p}$ with $i_{1} \geq p$ and $p=i_{2} \leq i_{3} \leq \cdots \leq i_{n}$.

Since $r$ is split by the map $i$ from the pushout description of $X(k)$, the induced map $r_{*}$ takes the basis elements $(i)_{p}$ to give a basis of $E(1)_{2 p, *}^{2}$. Since the spectral sequences collapse at $E^{1}$, and since the $E^{1}$ terms are free over $E_{A}^{*}$, it follows that the basis elements of $E_{*}^{A}\left(\mathbb{C} P(\mathcal{U}) \times G r_{n-1}(\mathcal{U})\right)$ giving rise to the basis elements $(i)_{p}$ for some $p$ (namely those with $i_{1} \leq i_{2} \leq \cdots \leq i_{n}$ ) map to a basis of $E_{*}^{A}\left(G r_{n}(\mathcal{U})\right)$ as required.

Remark 5.8. The referee suggests the following attractive repackaging of the results of this section. For any representation $W$, we can define a spectrum

$$
R_{W}:=\bigvee_{k \geq 0} G r_{k}(\mathcal{U})^{\operatorname{Hom}(\gamma, W)} .
$$

Direct sum makes this into a ring spectrum and indeed there is a strictly commutative ring spectrum in the category of [5] weakly equivalent to $R_{W}$ : one takes the spectrum associated to the pre-spectrum whose $V$ th space is $\bigvee_{k} \Sigma^{V} \operatorname{Gr}_{k}(V)^{\operatorname{Hom}(\gamma, W)}$.

The construction is natural in the sense that if $V \subseteq W$, the inclusion of Thom spaces gives a ring map $R_{V} \longrightarrow R_{W}$. The particular case $V=0$ gives a cofibre sequence

$$
J_{W} \longrightarrow R_{0} \longrightarrow R_{W}
$$

Theorem 4.1 then states

$$
J_{V^{k}} \simeq \bigvee_{n} G r_{n}(\mathcal{U})^{[k-1]} .
$$


A one dimensional representation $\alpha$ gives a map

$$
i_{\alpha}: S^{\operatorname{Hom}(\alpha, W)} \longrightarrow G r_{1}(\mathcal{U})^{\operatorname{Hom}(\gamma, W)} \subseteq R_{W}
$$

By Lemma 4.5 there is a cofibre sequence

$$
S^{\operatorname{Hom}(\alpha, W)} \wedge R_{W} \stackrel{i_{\alpha}}{\longrightarrow} R_{W} \longrightarrow R_{W \oplus \alpha},
$$

so that in particular

$$
J_{W \oplus \alpha} / J_{W} \simeq \operatorname{fibre}\left(R_{W} \longrightarrow R_{W \oplus \alpha}\right) \simeq S^{\operatorname{Hom}(\alpha, W)} \wedge R_{W} .
$$

Now for a complex orientable theory we may package the results of this section as saying

$$
E_{*}^{A}\left(R_{0}\right)=\operatorname{Symm}\left(E_{*}^{A}(\mathbb{C} P(\mathcal{U}))\right),
$$

and more generally

$$
E_{*}^{A}\left(R_{W}\right)=\operatorname{Symm}\left(E_{*}^{A}(\mathbb{C} P(\mathcal{U}), \mathbb{C} P(W))\right) .
$$

\section{Thom classes}

It follows from what we have proved that any complex oriented cohomology theory has Thom classes for arbitrary bundles. It is the purpose of the present section to make this explicit: we prove Theorem 1.1.

Following Okonek [7] we say that $E_{A}^{*}(\cdot)$ has Thom classes if for any complex vector bundle over a space $X$, there is an element $\tau(\xi) \in E_{A}^{*}\left(X^{\xi}\right)$ so that this system of elements is

1. natural for bundle maps,

2. product preserving in the sense that $\tau(\xi \times \eta)=\tau(\xi) \wedge \tau(\eta)$ and

3. normalized so that if $X=A / B$ and the fibre of $\xi$ over $B$ is the representation $W$ of $B$ then $\tau(\xi)=\sigma_{V}(1)$

Remark 6.1. (i) Okonek only requires the normalization when $B=A$.

(ii) To obtain Thom isomorphisms we would only require the normalization that $\tau(\xi)$ was a unit multiple of $\sigma_{W}(1)$. However, we show that any complex oriented cohomology theory has Thom classes in the present stricter sense.

To prove that a system of Thom classes gives Thom isomorphisms

$$
E_{A}^{*}(X) \stackrel{\cong}{\longrightarrow} \tilde{E}_{A}^{*}\left(X^{\xi}\right)
$$

(essentially by cup product with $\tau(\xi)$ ) or

$$
\tilde{E}_{*}^{A}\left(X^{\xi}\right) \stackrel{\cong}{\longrightarrow} E_{*}^{A}(X)
$$


(essentially by cap product with $\tau(\xi)$ ) we proceed from the fact that $\sigma_{V}$ is an isomorphism. We may then work our way up the cellular filtration of $X$ using the normalization condition. Alternatively, if $\xi$ has a complement $\eta$ so that $\xi \oplus \eta=V$ we may use the product preserving property.

Before proceeding we make a reduction.

Lemma 6.2. A cohomology theory $E_{A}^{*}(\cdot)$ has a system of Thom classes if and only if it has universal Thom classes,

$$
\tau_{n} \in \tilde{E}_{A}^{*}\left(G r_{n}(\mathcal{U})^{\gamma_{n}}\right)
$$

for each $n$, so that

1. the system is product preserving in the sense that under the direct sum map $G r_{m}(\mathcal{U}) \times G r_{n}(\mathcal{U}) \longrightarrow G r_{m+n}(\mathcal{U})$, the class $\tau_{m+n}$ pulls back to $\tau_{m} \wedge \tau_{n}$

2. Over the point $W$ of $G r_{n}(\mathcal{U})$ with isotropy $B \subseteq A$ the class $\tau_{n}$ restricts to $\sigma_{W}(1)$;

We may then define the Thom class for an n-plane bundle by pulling back $\tau_{n}$ under the Thomification of its classifying map.

Theorem 6.3. If $E$ is a complex oriented theory with orientation in cohomological degree 2 and complex stable structure determined by the orientation, there is an associated system of Thom classes.

Proof. We shall construct classes $\tau_{n}$ as specified in 6.2. Assume that the flag begins with $V^{1}=\epsilon$. By pullback along conjugation, we see that

$$
G r_{n}(\mathcal{U})^{\gamma_{n}} \cong G r_{n}(\mathcal{U})^{\gamma_{n}^{*}} \simeq G r_{n}(\mathcal{U}) / G r_{n}(\mathcal{U})^{[0]},
$$

and we have identified the cohomology of this space exactly. From the orientation we construct the cohomology class $y(\epsilon) \in E_{A}^{*}(\mathbb{C} P(\mathcal{U}))$, and hence by 2.2 , the element $y(\epsilon)^{\otimes n} \in E_{A}^{*}\left(G r_{n}(\mathcal{U})\right)$. By construction this restricts to zero in $E_{A}^{*}\left(G r_{n}(\mathcal{U})^{[0]}\right)$, and thus we obtain

$$
\tau_{n}=y(\epsilon)^{\otimes n} \in E_{A}^{*}\left(G r_{n}(\mathcal{U}), G r_{n}(\mathcal{U})^{[0]}\right) \cong \tilde{E}_{A}^{*}\left(G r_{n}(\mathcal{U})^{\gamma_{n}}\right) .
$$

The element $\tau_{n}$ is identified with a map

$$
\tau_{n}: G r_{n}(\mathcal{U})^{\gamma_{n}} \longrightarrow E .
$$

Lemma 6.4. The classes $\tau_{n}$ are compatible under product in the sense that

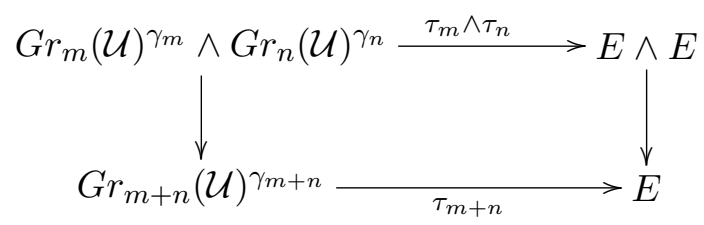

commutes up to homotopy. 
Proof. This follows from the fact that $y(\epsilon)^{\otimes m} \otimes y(\epsilon)^{\otimes n}=y(\epsilon)^{\otimes(m+n)}$.

Lemma 6.5. The class $\tau_{n}$ is a Thom class for $\gamma_{n}$.

Proof. An $A$-fixed point of $G r_{n}(\mathcal{U})$ is a representation $V=\beta_{1} \oplus \beta_{2} \oplus$ $\cdots \oplus \beta_{n}$, and such a point lies in the image of the direct sum map from $(\mathbb{C} P(\mathcal{U}))^{\times n}$. By naturality it therefore suffices to deal with the case $n=1$. However, by definition of an orientation $\tau_{1}=y(\epsilon)$ restricts to a generator of $E_{A}^{*}(\mathbb{C} P(\alpha \oplus \epsilon), \mathbb{C} P(\epsilon))$. Since we have used the orientation to give the complex stable structure, the generator is $\sigma_{\alpha^{-1}}(1)$ as required.

For the fibre over a non-fixed point we use the fact that orientations behave well under restriction to subgroups.

\section{Universality of complex cobordism}

Let us turn now to the spectrum level statements, and the connection with maps $M U \longrightarrow E$ : we prove Theorem 1.2.

First we deduce that the existence of a ring map $M U \longrightarrow E$ gives $E$ a complex orientation. This is not quite obvious because of the distinction between a map of spectra and a map preserving the complex stable structure.

Lemma 7.1. If there is a ring map $\theta: M U \longrightarrow F$ of $A$-spectra, then $F$ is complex orientable, and the image of the canonical orientation of $M U$ is an orientation of $F$.

Remark 7.2. The proof makes no special use is made of the fact that the domain is $M U$ or the fact that we use its canonical orientation. However our results show that the general case follows from this one.

Proof. Let $x(\epsilon) \in \tilde{M} U^{*}(\mathbb{C} P(\mathcal{U}))$ denote an orientation of $M U$. By hypothesis the restriction $\lambda\left(\alpha^{-1}\right)$ of $x(\epsilon)$ to $\tilde{M} U^{*}\left(S^{\alpha^{-1}}\right)$ is a generator as an $M U_{A^{-}}^{*}$ module. From the suspension isomorphism $\tilde{M} U^{*}\left(S^{\alpha^{-1}}\right) \cong \tilde{M} U^{*-\alpha^{-1}}$, and $\lambda\left(\alpha^{-1}\right)$ is a unit in the $R O(G)$-graded coefficient ring. Hence $\theta_{*}\left(\lambda\left(\alpha^{-1}\right)\right)$ is also a unit in the $R O(G)$-graded sense, and therefore $\theta_{*}(x(\epsilon))$ restricts to a generator of the integer graded module $\tilde{E}_{A}^{*}\left(S^{\alpha^{-1}}\right)$ as required.

In Sect. 6 we showed that any complex oriented theory has Thom classes for arbitrary bundles. To complete the proof of 1.2 it therefore remains only to construct a map $M U \longrightarrow E$ from a system of Thom classes.

First recall that $M U$ is the prespectrum given on a subspace $V \subset \mathcal{U}$ of dimension $n$ by

$$
M U(V)=G r_{n}(V \oplus \mathcal{U})^{\gamma_{n}}
$$


If $U \subseteq V$ with $U$ of dimension $m$, the structure map

$$
S^{V-U} \wedge M U(U)=G r_{m}(U \oplus \mathcal{U})^{(V-U) \oplus \gamma_{m}} \longrightarrow G r_{n}(V \oplus \mathcal{U})^{\gamma_{n}}
$$

is given by Thomifying the map adding $V-U$. Thus

$$
M U \simeq \operatorname{holim}_{\rightarrow} \Sigma^{-V} G r_{n}(V \oplus \mathcal{U})^{\gamma_{n}}
$$

and the cohomology of $M U$ may be calculated from that of Thom spaces of Grassmannians by the Milnor exact sequence.

It is useful to be more explicit about the homology.

Lemma 7.3. Provided the complex stable structure is defined by the orientation, the structure map $\Sigma^{V-U} G r_{n}(U \oplus \mathcal{U})^{\gamma_{m}} \longrightarrow G r_{n}(V \oplus \mathcal{U})^{\gamma_{n}}$, takes the element

$$
\sigma_{V-U} \tau_{m}\left(\beta_{i_{1}}(\mathcal{F}) \otimes \beta_{i_{2}}(\mathcal{F}) \otimes \cdots \otimes \beta_{i_{m}}(\mathcal{F})\right)
$$

to

$$
\left.\tau_{n}\left(\beta_{0}(\mathcal{F}) \otimes \cdots \otimes \beta_{0}(\mathcal{F}) \otimes \beta_{i_{1}}(\mathcal{F})\right) \otimes \beta_{i_{2}}(\mathcal{F}) \otimes \cdots \otimes \beta_{i_{m}}(\mathcal{F})\right) .
$$

Proposition 7.4. The classes $\tau_{n}$ assemble to give a unique map

$$
\tau: M U \longrightarrow E
$$

of ring spectra.

Proof. Since the structure maps are surjective in cohomology,

$$
E_{A}^{*}(M U)=\lim _{\leftarrow V} E_{A}^{*}\left(\Sigma^{-V} G r_{n}(\mathcal{U})^{\gamma_{n}}\right) .
$$

The existence of the map $\tau$ therefore follows from compatibility of the elements $\tau_{n}$ under suspension.

Lemma 7.5. The classes $\tau_{n}$ are compatible under suspension in the sense that

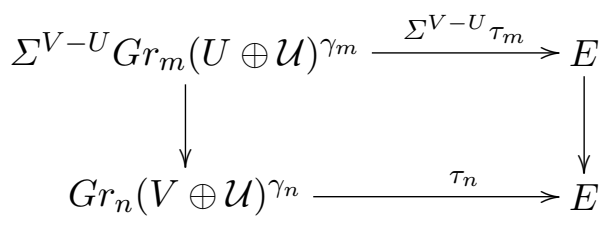

commutes up to homotopy.

Proof. First note that the space of isometric isomorphisms $\mathcal{U} \longrightarrow \mathcal{U}$ is contractible, so the particular identification $V \oplus \mathcal{U} \cong \mathcal{U}$ is not important. Now, since the structure maps in $M U$ arise from bundles, the lemma follows from 6.4.

Since the smash product $M U \wedge M U \longrightarrow M U$ is induced by the Thomification of the map classifying direct sum of bundles, the compatibility of the elements $\tau_{n}$ under products shows that the map $\tau$ is a map of ring spectra. This completes the proof of 1.2. 


\section{Homology and cohomology of $M U$}

In this section we give an account of the relationship between orientations and ring maps $M U \longrightarrow E$ which makes no explicit reference to the orientation. The main new result is Theorem 1.3.

First we need to calculate the homology of $M U$. We use the notation of Sect. 7. Of course we have

$$
\begin{aligned}
E_{*}^{A}(M U) & =\lim _{\rightarrow n} E_{*}^{A}\left(\Sigma^{-V^{n}} M U\left(V^{n}\right)\right) \\
& =\lim _{\rightarrow n} E_{*}^{A}\left(\Sigma^{-V^{n}} G r_{n}\left(V^{n} \oplus \mathcal{U}\right)^{\gamma_{n}}\right) .
\end{aligned}
$$

Thus we may construct elements using the maps

$$
\begin{aligned}
E_{*}^{A}\left(G r_{n}(\mathcal{U})\right) & \cong E_{*}^{A}\left(\Sigma^{-V^{n}} G r_{n}(\mathcal{U})^{\gamma_{n}}\right) \\
& =E_{*}^{A}\left(\Sigma^{-V^{n}} M U\left(V^{n}\right)\right) \longrightarrow E_{*}^{A}(M U) .
\end{aligned}
$$

We identified the effect of the maps in the direct system in 7.3. It is natural to suppress the terms $\beta\left(V^{0}\right)$.

Definition 8.1. We write $b_{i}(\mathcal{F})$ for the image of $\beta_{i+1}(\mathcal{F})$ in $E_{*}^{A}(M U)$. It follows that the image of $\beta_{i_{1}+1}(\mathcal{F}) \otimes \beta_{i_{2}+1}(\mathcal{F}) \otimes \cdots \otimes \beta_{i_{n}+1}(\mathcal{F})$ is $b_{i_{1}}(\mathcal{F}) b_{i_{2}}(\mathcal{F}) \cdots b_{i_{n}}(\mathcal{F})$. In the nonequivariant context, it is standard to write $\beta_{i+1}$ for $\beta_{i+1}(\mathcal{F})$ and $b_{i}$ for $b_{i}(\mathcal{F})$.

Note that Whitney sum of bundles gives a product $M U \wedge M U \longrightarrow M U$, and hence $E_{*}^{A}(M U)$ is an $E_{A}^{*}$-algebra. The simplicity of the following theorem is somewhat surprising.

Theorem 8.2. If $E_{A}^{*}(\cdot)$ is a complex oriented theory, the homology of $M U$ is polynomial over $E_{A}^{*}$ :

$$
E_{*}^{A}(M U)=E_{A}^{*}\left[b_{1}(\mathcal{F}), b_{2}(\mathcal{F}), b_{3}(\mathcal{F}), \cdots\right] .
$$

Furthermore there is a Künneth theorem in the sense that $E_{A}^{*}\left(M U^{\wedge s}\right)=$ $E_{A}^{*}(M U)^{\otimes s}$.

Proof. From our construction of the generators of the homology of Grassmannians, their behaviour under Whitney sum is obvious. The result for $M U$ follows from the relation $(\sigma \otimes \tau) \cap(\beta \otimes \gamma)= \pm(\sigma \cap \beta) \otimes(\tau \cap \gamma)$.

It is now straightforward to deduce the global statements we require. The additive part is no problem since the homology of $M U$ is free over $E_{A}^{*}$, and we have a Künneth isomorphism. 
Corollary 8.3. If E is complex orientable, passage to E-homology gives

$$
\left[M U^{\wedge s}, E\right]_{*}^{A}=E_{*}^{A}-\bmod \left(E_{*}^{A}\left(M U^{\wedge s}\right), E_{*}^{A}\right) .
$$

We really want to understand the set $\operatorname{Ring}_{A}[M U, E]$ of homotopy classes of ring maps $M U \longrightarrow E$. Since $E_{*}^{A}(M U)$ is a free $E_{*}^{A}$-algebra, this too is immediate.

Corollary 8.4. If E is complex orientable, passage to E-homology gives

$$
\operatorname{Ring}[M U, E]^{A}=E_{*}^{A}-\operatorname{alg}\left(E_{*}^{A}(M U), E_{*}^{A}\right) .
$$

Combining this with 7.4 and being careful about normalization, we obtain a useful consequence.

Corollary 8.5. If $x(\epsilon)$ is a complex orientation of $E_{A}^{*}(\cdot)$, then there is a natural bijective correspondence between orientations of $E_{A}^{*}(\cdot)$ in cohomological degree 2 which give the same complex stable structure as $x(\epsilon)$ and

$$
\operatorname{Ring}[M U, E]^{A}=E_{*}^{A}-\operatorname{alg}\left(E_{*}^{A}(M U), E_{*}^{A}\right) .
$$

More precisely, suppose $x^{\prime}(\epsilon)$ is an orientation in cohomological degree 2 giving the same complex stable structure as $x(\epsilon)$. If $V^{1}=\epsilon$ and the associated parameter is

$$
y^{\prime}(\epsilon)=\Sigma_{i} \lambda_{i} y\left(V^{i}\right)
$$

then $\lambda_{0}=0$ and $\lambda_{1}=1$. The associated algebra homomorphism

$$
E_{*}^{A}(M U)=E_{A}^{*}\left[b_{1}(\mathcal{F}), b_{2}(\mathcal{F}), b_{3}(\mathcal{F}), \ldots\right] \longrightarrow E_{A}^{*}
$$

is then determined by

$$
b_{i}(\mathcal{F}) \longmapsto \lambda_{i+1}
$$

for $i \geq 1$.

Proof. In general, if $x^{\prime}(\epsilon)$ is an orientation of $E$, then the associated map $M U \longrightarrow E$ restricts to $x^{\prime}(\epsilon)^{\otimes n}$ as a map

$$
\Sigma^{-2 n}\left(\mathbb{C} P(\mathcal{U})^{\times n}\right)^{\gamma_{n}} \longrightarrow \Sigma^{-2 n}\left(G r_{n}(\mathcal{U})\right)^{\gamma_{n}} \longrightarrow E .
$$

Thus the algebra homomorphism associated to $x^{\prime}(\epsilon)$ may be calculated on an element $z$ arising from $\Sigma^{-2 n} M U(V)$ as $x^{\prime}(\epsilon)^{\otimes n}(z)$.

Now suppose $x^{\prime}(\epsilon)$ is expressed in terms of the basis associated to the orientation $x(\epsilon)$. The coefficient $\lambda_{0}=0$ since $y^{\prime}(\epsilon)$ restricts trivially to $\mathbb{C} P(\epsilon)$. The coefficient $\lambda_{1}$ is obtained by restricting to $\left(\mathbb{C} P\left(V^{2}\right), \mathbb{C} P\left(V^{1}\right)\right) \cong S^{\alpha_{2}^{-1}}$; since the complex stable structures defined by $x(\epsilon)$ and $x^{\prime}(\epsilon)$ agree, the coefficient is 1 . It is easy to see the images of $b_{i}(\mathcal{F})$ for $i \geq 1$ are as specified since they arise from $\Sigma^{-V^{1}} M U\left(V^{1}\right)$. 
Remark 8.6. (i) For a complex stable structure arising from an orientation, the element $\sigma_{\alpha}(1)$ for any chosen $\alpha$ determines the rest, because of the action of $A^{*}$. This explains why consideration of $\alpha_{2}^{-1}$ alone was enough to show $\lambda_{1}=1$.

(ii) The statement of the corollary is consistent with the conjecture that $M U_{*}^{A} M U$ classifies strict isomorphisms of $A$-equivariant formal group laws.

\section{References}

1. J.F.Adams, Stable homotopy and generalized homology. Chicago University Press (1974)

2. M.Cole, Complex oriented $R O(G)$-graded equivariant cohomology theories and their formal group laws. Thesis, University of Chicago (1996)

3. M.Cole, Complex oriented equivariant cohomology theories and equivariant complex projective spaces (In preparation)

4. Michael Cole, J.P.C.Greenlees and I.Kriz, Equivariant formal group laws. Proc. London Math. Soc 81 (2000) 355-386

5. A.D.Elmendorf, I.Kriz, M.A. Mandell and J.P.May, Rings, modules and algebras in stable homotopy theory. American Mathematical Society Surveys and Monographs, American Mathematical Society, (1997)

6. J.W.Milnor and J.Stasheff, Characteristic classes. Princeton University Press (1974)

7. C.Okonek, Der Conner-Floyd-Isomorphismus für Abelsche Gruppen. Math. Z. 179 (1982) 201-212 